\title{
Bilateral Temporal Bone Fractures with Bilateral Facial Nerve Palsy
}

\author{
Kanjully Sabarigirish ${ }^{1}$ Lohith B.R. ${ }^{1} \quad$ Sanjeev Saxena ${ }^{1}$ Himanshu Swami ${ }^{1}$ Angshuman Dutta ${ }^{1}$ \\ ${ }^{1}$ Department of Otorhinolaryngology, Command Hospital Air Force \\ Bangalore, Rajiv Gandhi University of Health Sciences, Bangalore, \\ Karnataka, India

\begin{abstract}
Address for correspondence Lohith B.R., MS (ENT), Department of Otorhinolaryngology, Command Hospital Air Force Bangalore, Rajiv Gandhi University of Health Sciences, Bangalore, Karnataka 560007,
\end{abstract} \\ India (e-mail: lohithbr@gmail.com).
}

Indian J Neurotrauma 2016;13:168-170.

\begin{abstract}
Keywords

- bilateral facial palsy

- fracture

- temporal bone
\end{abstract}

\section{Introduction}

The temporal bone is a complex bone encapsulating many vital structures. Fracture of the temporal bone, especially when it violates the otic capsule, tend to injure facial nerve more than the fractures that spare the otic capsule. Most fractures of the temporal bone are unilateral and otic capsule sparing. Bilateral temporal bone fracture with facial nerve injury is a rarity. Involvement of both facial nerves augments the diagnostic and therapeutic challenges involved.

\section{Case Report}

A 27-year-old man was referred with the inability to close both the eyes completely from the neurosurgical department. It followed a road traffic accident 10 days prior, resulting in closed head injury and intracranial bleeding, which was managed with appropriate neurosurgical intervention. Physical examination revealed an expressionless countenance and complete immobility of all facial muscles with HouseBrackmann grading of $6 / 6$. On closure of the eyes, Bell's phenomenon was observed on both sides ( $\mathbf{- F i g . ~ 1 ) . ~ O t o s c o p y ~}$ revealed bilateral hemotympanum, but showed no evidence of injury of external auditory canal or cerebrospinal fluidotorrhea.
Audiological evaluation confirmed bilateral conductive hearing loss and bilateral flat type B curve in tympanometry. Electroneuronography (ENoG) showed reduction in amplitude of the waveform on both sides on maximal stimulation. Highresolution computed tomography scan of the temporal bone revealed symmetrical longitudinal fractures coursing through the petrous part, sparing the otic capsules but extending cranially to the squamous part. On the both sides, the fracture line crossed the region of the geniculate ganglia of the seventh

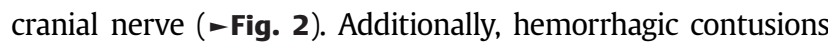
were observed on both temporal and parietal lobes, with focal subarachnoid hemorrhage over the right temporal lobe.

The patient underwent surgical intervention of both sides on the 12th day after onset. On the right side, facial nerve transection was observed on the proximal part of the horizontal segment adjoining the geniculate ganglion. A short segment of greater auricular nerve was used for neural grafting. Limited access to the proximal end of the transsection prevented the graft from being secured with sutures to the nerve. The grafted segment was therefore wrapped around in temporalis facia and stabilized with fibrin glue. On the left side, there was no discontinuity of the nerve, but nerve sheath was edematous in the horizontal segment, which was duly decompressed. Follow-up 6 months after surgery revealed 


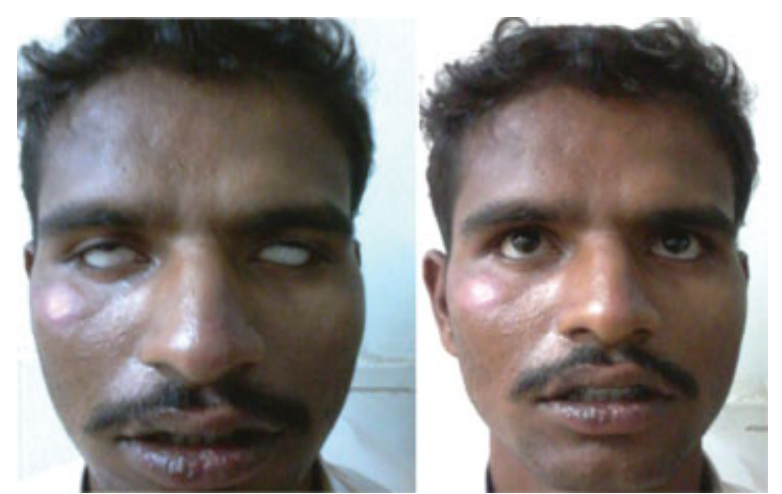

Fig. 1 Bell's phenomenon and masklike expression in bilateral facial nerve palsy.

House-Brackmann grading of 5/6 on the left side and $3 / 6$ on the right side.

\section{Discussion}

Bilateral facial nerve palsy, defined as involvement of both hemifacies within 4 weeks of onset, is a rare condition occurring in $0.4 \%$ of all cases of facial nerve palsy. ${ }^{1}$ The commonest cause, Bell's palsy, is a diagnosis arrived by exclusion of traumatic, metabolic, immunological, infective, and vascular causes. ${ }^{2}$ Fractures of skull base occur in 4 to $30 \%$ of closed head injuries due to falls, assault, or traffic accidents, with temporal bones involved in $22 \%{ }^{3}$ These fractures are mostly unilateral, with bilateral fractures reported in less than $20 \%$. Of these, bilateral facial nerve palsy is limited to a mere $3 \%{ }^{4}$

Conventionally temporal bone fractures are described as longitudinal or transverse based on its alignment to the axes of the temporal bone. However, descriptors such as oblique fractures have been added to describe a fracture plane that crosses the petrotympanic fissure. ${ }^{5}$ Longitudinal fractures are most likely to involve the fallopian canal in the region of the geniculum, whereas labyrinthine segment is the usual site of injury in transverse fractures. Transection of the nerve is more common in transverse fractures. ${ }^{6}$ These morphological
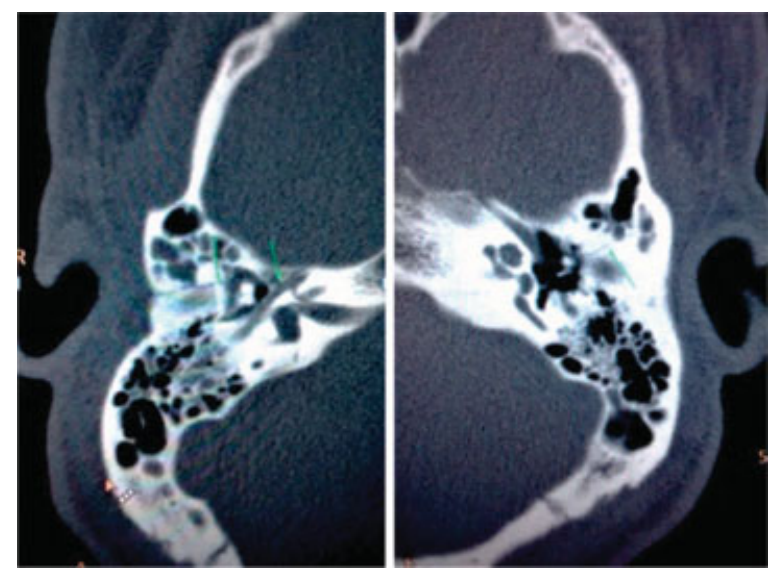

Fig. 2 Symmetrical bilateral temporal bone fractures involving the geniculate ganglion. descriptions of the fractures do not accurately correlate with the clinical sequelae. Involvement of the otic capsule is a more accurate predictor of the outcome. Facial nerve injury is twice as common when the otic capsule is violated. ${ }^{7}$ High-resolution imaging of the temporal bone plays a crucial role in accurately identifying the site of injury. However, assessing the severity of damage with certainty, especially when a transection of the nerve is suspected, is often difficult radiologically.

Electrophysiological tests such as ENoG have the potential to assess the severity of neuronal damage and degeneration with precision. When done serially in the window period between 3 and 21 days after onset, it determines the threshold for surgical intervention when $90 \%$ or more of neuronal degeneration is recorded. However, use of ENoG is compromised in bilateral facial nerve palsy as ENoG can only determine the degree of neuronal degeneration when the evoked amplitude of the traumatized side is compared with that of the unaffected side in unilateral facial nerve palsy.

Therefore, in posttraumatic bilateral facial nerve paralysis, decision on the necessity of surgical intervention and its timing is primarily based on the immediacy of onset and completeness of palsy. Immediate complete palsy indicates loss of neural discontinuity, whereas delayed onset or partial palsy suggests an intact nerve compromised by neural edema and/or hematoma in the fallopian canal compressing the nerve. Often, an early diagnosis of bilateral facial nerve palsy secondary to head injury is delayed as the nerve function cannot be checked in an unresponsive comatose patient. A masklike facial expression with lack of asymmetry of the face on volitional movement adds to the diagnostic dilemma. ${ }^{8}$ Surgical intervention also tend to get delayed due to unfavorable posttraumatic neurologic status. However, inappropriate delay should not be the aver surgical intervention as studies suggest reasonable recovery even in surgical intervention performed as late as 160 days. ${ }^{9,10}$

\section{Conclusion}

Bilateral temporal bone fracture with bilateral facial nerve palsy is a rare entity. Clinical diagnosis of this condition is often delayed in a neurologically compromised patient. Highresolution imaging accurately identifies the site of facial nerve injury but not the severity of the injury. ENoG, a useful tool for assessing neuronal degeneration in unilateral facial nerve palsy, is not feasible in bilateral involvement of facial nerve. Immediate surgical intervention is warranted when there is immediate, complete posttraumatic facial nerve palsy.

\section{References}

1 Kim YH, Choi IJ, Kim HM, Ban JH, Cho CH, Ahn JH. Bilateral simultaneous facial nerve palsy: clinical analysis in seven cases. Otol Neurotol 2008;29(3):397-400

2 Ghiasi S, Banaei M. Bilateral facial paralysis caused by temporal bone fracture: a case report. Arch Trauma Res 2016;5(2):e26892

3 Li J, Goldberg G, Munin MC, Wagner A, Zafonte R. Post-traumatic bilateral facial palsy: a case report and literature review. Brain Inj 2004;18(3):315-320 
4 Cannon CR, Jahrsdoerfer RA. Temporal bone fractures. Review of 90 cases. Arch Otolaryngol 1983;109(5):285-288

5 Ghorayeb BY, Yeakley JW. Temporal bone fractures: longitudinal or oblique? The case for oblique temporal bone fractures. Laryngoscope 1992;102(2):129-134

6 Wysocki J. Cadaveric dissections based on observations of injuries to the temporal bone structures following head trauma. Skull Base 2005;15(2):99-106, discussion 106-107

7 Dahiya R, Keller JD, Litofsky NS, Bankey PE, Bonassar LJ, Megerian CA. Temporal bone fractures: otic capsule sparing versus otic capsule violating clinical and radiographic considerations. J Trauma 1999;47(6):1079-1083

8 Kumar R, Mittal RS. Post traumatic delayed bilateral facial nerve palsy (FNP): diagnostic dilemma of expressionless face. J Clin Diagn Res 2015;9(4):PD15-PD16

9 Sanuş GZ, Tanriöver N, Tanriverdi T, Uzan M, Akar Z. Late decompression in patients with acute facial nerve paralysis after temporal bone fracture. Turk Neurosurg 2007;17(1):7-12

10 Quaranta A, Campobasso G, Piazza F, Quaranta N, Salonna I. Facial nerve paralysis in temporal bone fractures: outcomes after late decompression surgery. Acta Otolaryngol 2001;121(5):652-655 